\title{
Educational and ethical considerations
}

\section{Creating a patient-led NHS: some ethical and epistemological challenges}

\author{
John Owens \\ Centre for Public Policy Research, King's College London, UK
}

\section{Key messages}

- The Coalition government's recent Open Public Services White Paper states their intention to give patients direct control over healthcare services wherever possible, primarily by offering patents greater opportunity to participate in and make choices about the care they receive.

- The creation of a so called 'patient-led NHS' requires the NHS to accommodate and respond to the beliefs, values and agendas of patients as well as those of medical professionals. Since it cannot be assumed that patients will routinely share the beliefs and values of medical professionals, a shift towards greater patient autonomy may lead to increased levels of conflict within the processes of consultation and commissioning.

- Consequently, it should be recognised that a complex set of ethical and epistemological challenges accompany the proposals to create a patient- led NHS, particularly surrounding the balance between paternalism and autonomy in the doctor-patient relationship and the identification of the appropriate ends and means of treatment in the consultation and commissioning processes.

- Successful implementation of these reforms will require a greater awareness of the nature of these issues as well as further research into how these challenges might best be met.

\section{Why this matters to me}

In 2010 I completed my $\mathrm{PhD}$ in applied philosophy which considered personalisation of the NHS from a philosophical perspective, concentrating in particular on the epistemic and ethical issues involved in granting patients greater opportunity to participate and make choices about their care.

\section{ABSTRACT}

This article responds to the Coalition government's recent Open Public Services White Paper and to proposals which call for the creation of a 'patientled NHS' which will, wherever possible, seek to give patients direct control over the services they receive, through a greater degree of choice and participation. Its central contention is that affording patients greater influence over the consultation and commissioning processes will require the NHS to accommodate and respond to the beliefs, values and agendas of patients, as well as those of medical professionals and policy makers. Since it cannot be assumed that professionals and patients will share the same beliefs, values and agendas, the creation of a system of patient-led services has the potential to bring disagreement between professionals and patients to a head, particularly within the consultation and commissioning processes. Thus, a set of complex epistemic and ethical challenges accompanies the proposed creation of a 'patient-led NHS', greater awareness of which will be necessary for the successful implementation of such reforms.

Keywords: commissioning, concordance, co-production, epistemology, ethics, NHS, patient-led, personalisation, shared decision making 


\section{Introduction}

The Coalition government's recent Open Public Services White Paper alongside their proposals for changing the NHS's commissioning structure, states a desire to hand greater control over the planning and delivery of healthcare services to patients. ${ }^{1,2}$ These proposals can be seen to reflect a wider ambition, shared with the previous Labour government, to create a 'patient-led NHS' in which patients have a greater opportunity to actively participate in the decisions that are made about their treatment and make choices about the services they receive. ${ }^{3}$ This article responds to these proposals, considering in particular what they might mean for the future of primary care for patients with long-term and chronic conditions.

Much of the popular criticism of the proposed changes to the NHS's commissioning structure has focused on the potentially harmful impact that greater competition and marketisation may have on the NHS, particularly insofar as this may affect access to care and levels of health inequality. In addition, a great deal has also been said about the effects of importing aspects of consumerist culture into healthcare practice and the potentially harmful impact this may have on professional-patient relationship through the erosion of traditional norms and values and as a consequence of the general pratitioner's (GP) role as patient advocate being undermined by their increased responsibility to marshal resources. ${ }^{4-7}$ Although these criticisms deserve serious attention, in this article, I wish to put them to one side and concentrate instead on some of the difficulties that may arise from the potential disagreement that may occur between medical professionals and patients in scenarios in which the latter are being encouraged to assert their own beliefs, values and agendas in the consultation and commissioning processes. This article seeks to draw attention to the potential epistemological and ethical difficulties that may be generated by transition to a patient-led NHS which must accommodate and respond to the patient's beliefs, values and agendas alongside those of medical professionals and policy makers. For instance, I consider the potential for epistemological conflict to occur within the course of a medical consultation should the doctor and patient disagree about the appropriate ends and means of treatment. Equally importantly, the shift towards a patient-led NHS underlines the importance of the ongoing ethical debate concerning the appropriate balance between patient autonomy and professional paternalism in the doctor-patient relationship, and I shall touch on this area too.

I suggest that greater awareness of these epistemological and ethical challenges - their nature, significance and the way in which they might be managed will be required if the creation of a patient-led NHS is to produce meaningful reform which avoids a potential scenario in which the consultation and commissioning processes have become fields for running battles between doctors and patients. However, this article is not meant as a constructive piece that offers a set of specific practical recommendations, rather I offer it as a critical reflection upon the proposed reforms. My goal is to enlighten the reader to some of the potential problems associated with the implementation of these reforms and if I succeed in raising awareness of these and stimulating debate among professionals and policy makers as to how they may be managed the article will have served its purpose.

\section{Creating a 'patient-led NHS'}

Much of the recent discourse and debate surrounding the reorganisation of the NHS has centred on the concept of 'personalisation'. The drive for personalisation can be seen as the latest example of the changing medical culture in the UK, calling for a movement away from the sort of paternalistic arrangements in which deferential patients would accept decisions made by authoritarian professionals in favour of greater opportunity for patients to participate in and make choices about the way in which their care is planned, delivered and evaluated. Indeed, personalisation builds on a host of similar initiatives which have called for greater patient involvement in the healthcare process: in the fields of prescribing and medicine taking, the Royal Pharmaceutical Society of Great Britain (RPSGB) introduced the concept of concordance, the notion of a 'therapeutic alliance' has been developed in mental health and there has been a movement towards shared decision making and 'values based practice' in general practice more generally, as expressed within the Royal College of General Practitioner's curriculum. ${ }^{8,9}$

In 2005, the Department of Health published a report entitled 'Commissioning a Patient-Led NHS' which focused on 'creating a step-change in the way services are commissioned by front-line staff, to reflect patient choices'. ${ }^{3}$ Personalisation is often explained in contrast to the 'one-size-fits-all' models of public services which it aims to replace. Although the term has been most widely used to drive through changes in social care provision, in the arena of healthcare personalisation it focuses on providing services which are tailored to the specific circumstances and requirements of individual patients, with particular emphasis on making changes to the way services are funded and delivered to improve the quality of patient experience. The main mechanism for delivering personalisation involves increasing the opportunities that patients 
have to participate in the delivery of services, and in most instances this is cashed out in terms of granting patients greater opportunity to make choices and to offer feedback about various aspects of the services they receive. Patient choice can be seen as occurring over the various elements of healthcare services in different ways and at different depths. For example, extending general practice opening hours has extended patients greater choice about the time they see their doctor, while the recently established right that patients have to select which hospital they attend for their elective surgery allows them to decide who it is that will provide the services they are entitled to. More radically, recent announcements suggest that the choices extended to patients will be deepened in a way that allows them to decide upon the content and means of delivery of the services they receive. That is, patients would be granted choice over the 'what', in addition to the 'when' and the 'who' of health services. In its recent Open Public Services White Paper, the Coalition government pledged that, wherever possible, choice for patients will be increased, describing this as the first principle of their public services reform agenda:

Our vision is for public services that revolve around each of us. That means putting people in control, either through direct payments, personal budgets, entitlements or choices. Wherever possible, we will increase choice by giving people direct control over the services they use. ${ }^{1}$

Personal budgets and direct payments have been widely introduced for users of social care services and, pending the results of ongoing pilot schemes, looks set to be introduced in certain areas of the NHS, especially for suffers of long-term and chronic conditions. Direct payments involve taking funding which has formally been allocated to central institutions and distributing it to patients who will then be able to purchase services from providers directly. While the direct payments scheme provides perhaps the most radical example of the potential patients may have to exercise choice, the personalisation movement more generally emphasises the importance of patient participation and enhanced autonomy. Indeed, calls for 'patient-centred care' and 'shared decision making' can be seen to fall within the bracket of personalisation. ${ }^{10}$ Moreover, the wider proposed reform of the NHS's commissioning structure, from a centralised model run by primary care trusts (PCTs) to one based on GP-led clinical commissioning groups appear to be in part motivated by a desire to bring the decision-making process closer to patients, allowing them, through dialogue with their physician, greater influence over the way money is spent.

\section{Epistemological and ethical challenges}

It remains to be seen exactly what these proposals will mean in practice for the NHS's operational and organisational culture. Indeed, as has been made clear elsewhere, personalisation is a vague term which could deliver a range of different outcomes. ${ }^{10}$ However, enactment of the more ambitious elements of personalisation has the potential to significantly alter the operational and cultural architecture of the NHS, from the dynamics of the doctor-patient relationship and the management of the consultation process, through to the various stages of commissioning, delivery and evaluation of services. For, at a fundamental level, calls for a patient-led NHS appear to demand the NHS to change from an institution which has traditionally been run according to the beliefs, values and agendas of medical professionals, managers and policy makers, to one which must accommodate and respond to the beliefs, values and agendas of lay patients.

At this point, I should make clear that I am certainly not objecting to greater patient autonomy per se, and indeed would agree with those who see greater patient voice and representation as an important and necessary element of good healthcare practice. Rather, my intention is to draw attention to the challenges that enhanced patient autonomy may pose on occasions when the patient holds different beliefs about the appropriate ends and means of the treatment process from those of the medical professional. Indeed, it seems that much of the literature presenting personalisation assumes that the creation of a patient-led NHS is hindered only by the typically limited opportunity that patients have had to participate, and that once mechanisms for supporting patient choice are introduced this goal will be relatively straightforward to achieve. However, offering patients greater opportunity to participate in the planning, delivery and evaluation of their treatment may lead to a situation in which disagreement between the doctor and patient - which has historically been submerged beneath a culture of lay deference to professional paternalism and an institutional architecture which favours professional control over decision making - rises to the surface. Thus, it may be that one consequence of the transition to a patient-led NHS is that the consultation and commissioning processes more frequently become sites for conflict between medical professional and patient.

Although it is perhaps easy to overstate the potential frequency and scope of such conflict, there are several reasons for taking it seriously. For example, research by both the RPSGB and the World Health Organization (WHO) suggests that approximately 
half of all patients fail to take medicines as prescribed, and ascribe this finding primarily to the health beliefs of the patient. ${ }^{8,11}$ Moreover, there exists a vast literature in the fields of bioethics and medical sociology which outlines the extent to which health may be conceived of according to starkly contrasting perspectives. For example, while figure like Boorse, Daniels and Groopman describe health in the technical terms of scientific biomedicine, writers like Canguilhem, Nordenfelt and Seedhouse characterise health very differently as a socially constructed category related to the well-being, capacities and experiences of the patient. ${ }^{12-17}$ Whereas the former perspective is based on secondary observation of the patient's condition according to the various disciplines of anatomy, physiology and pathology, the latter perspective has its roots in the direct phenomenological and social experience of the suffering patient who must learn to live with their illness. As I have explored in more detail elsewhere, the different relationships that doctors and patients have to the phenomenon of ill health provides reason to take seriously the suggestion that patients and medical professionals often do conceive of ill health in contrasting ways and, as a consequence, may hold different beliefs, values and agendas when it comes to decision making in the consultation and commissioning processes. ${ }^{18}$

So, while it would certainly be a crude simplification to assert that patients tend to hold one sort of perspective and medical professionals another, it does seem plausible that the background identities of patients and professionals and their relative relationships to the phenomenon of ill health itself may lead each party to hold different sorts of beliefs about the nature of the illness, the values and ends inherent in the treatment process and the best means of achieving these ends. And, importantly, once this potential difference has been recognised, it seems reasonable to suggest that one result of the creation of a patientled NHS will be a greater number of occasions when disagreements between patients and medical professionals are voiced.

To illustrate this point in more detail perhaps a closer look at what the movement towards a patientled NHS may mean for the commissioning of healthcare services will be useful. Essentially, commissioning is a process which involves the following four steps: identification of the patient's unsatisfied needs, identification of appropriate services which will lead to the satisfaction of these needs, procurement of services, and evaluation of the services' success in meeting the specified needs. The proposed reforms will hand patients a greater opportunity to participate in and exert an influence over each stage of this process by encouraging patients to articulate what they believe their needs to be, which services they believe to be suitable in the treatment of these needs as well as to say how effective these services have been. In particular, the introduction of direct payments would allow patients to make their own decisions about which resources and treatments are commissioned and which are not. As such, the tasks of identifying medical need, identifying an appropriate service to meet this need and evaluating the performance of this service would primarily be handed to the patient (albeit with support from the medical professional) and therefore subject to their own particular interpretation.

Accompanying scenarios in which greater patient choice leads to more frequent conflict between the patient and professional in primary care settings come associated ethical questions concerning how such conflict ought to be managed, most obviously those connected to the balance between of autonomy in the doctor-patient relationship. In connection with the notion of RPSGB's concordance Heath argues that if the proposals to increase patient autonomy are to amount to anything more than a rhetorical rebranding of the traditional paternalistic arrangements, medical professionals will have to yield to the increasing influence that patients will have in shaping NHS services. ${ }^{19}$ The Coalition government's Open Public Services White Paper states that 'people should be trusted to choose the best services for themselves rather than being forced to accept choices determined by others', however, little has been said about where the limits of the patient's power to choose ought to be set, nor according to which principles. ${ }^{1}$

Although the stated aim to increase patient autonomy suggests a willingness to accept and act upon the beliefs and values that underlie the patient's decisions, good practice in primary care will always involve striking a balance between promoting patient autonomy and ensuring that this choice is properly supervised in a way that does not compromise other important health goods. For example, Gawande and Loewy point to the need to balance patient autonomy with patient safety in order to ensure that the choices made by patients are appropriate for their condition and will not lead to greater harm. ${ }^{20,21}$ Clearly, conflict surrounding the ends and means of treatment between the patient and professional may extend to the concept of the patient's 'best interests' too, and the need to consider such matters represents a further point of complication in the ethical debate surrounding the creation of a patient-led NHS. In addition, it must be understood that the new opportunity that patients will be given to exercise choice will be set within the constraints of the institutional realities of the NHS which have typically provided a check on professional autonomy. These include the various economic, legal and professional factors, such as the NHS's limited financial resources, a concern to ensure that patient choice is extended equitably and in a way which is compatible with the wider interests of public 
health, as well as need to balance patient choice with the professional duty that doctors have to dissuade patients from making choices they consider to be inefficacious, inappropriate or even harmful. This last point shows the extent to which the ethical and epistemological challenges of creating a patient-led NHS are intertwined, since what counts as an efficacious, appropriate or safe treatment programme may be interpreted differently by professionals and patients.

\section{Conclusion}

Despite ongoing uncertainty surrounding exactly how the government's proposed reforms will be enacted in practice, the government's Open Public Services White Paper certainly suggest that patients will be granted a greater influence over the consultation and commissioning processes. The potential introduction of direct payments to patients suffering long-term and chronic conditions provides a radical opportunity for patients to direct the consultation and commissioning processes, requiring the NHS to accommodate the patient's beliefs, values and agendas alongside those of medical professionals and policy makers and within its existing institutional frameworks. I suggest that effective management of the potential problems involved in facilitating the transition to a patient-led NHS will require further research across a range of areas, but would like to finish by briefly highlighting the importance of including a philosophical contribution within this.

Although I have been unable to develop my analysis more fully here, Alan Cribb and I have outlined the benefits to be gained from developing a greater understanding of the relationship between the potentially divergent health beliefs of patients and professionals as well as an awareness of some of the epistemological and ethical challenges inherent in the creation of a patient-led NHS. ${ }^{18}$ Developing such understanding may be helpful since it highlights how the introduction of greater patient choice must be supported by a host of wider changes to the organisational, operational and institutional culture of the NHS and may also offer suggestions about how this may be achieved. For instance, recognition of the potential differences in health beliefs of professionals and patients suggests the need for a more reflexive medical culture which respects and is able to successfully manage potential conflict. One way to achieve this may be through greater emphasis on medical epistemology within the education of healthcare professionals, perhaps in a way which gives it a similar weight to the more established field of medical ethics. Another might be to stress the importance of providing professionals with adequate time and space to communicate with their patients in order to build up a deeper, more holistic understanding of their patients, including the beliefs and desires they hold towards the treatment of their condition.

\section{ETHICAL APPROVAL}

Not required

\section{CONFLICTS OF INTEREST}

None declared

\section{REFERENCES}

1 HM Government. Open Public Services White Paper. London: Cabinet Office, 2011.

2 Department of Health. Health and Social Care Bill. London: Department of Health, 2011.

3 Department of Health. Commissioning a Patient-Led NHS. London: Department of Health, 2005.

4 Clarke J and Newman J. What's in a name? - New Labour's citizen-consumers and the remaking of public services. Cultural Studies 2007;21(4).

$5 \mathrm{Mol} \mathrm{A}$. The Logic of Care: Health and the Problem of Patient Choice. London: Routledge, 2008.

6 Kmietowicz Z. Few doctors believe NHS reforms will improve quality of patient care, shows BMA survey. British Medical Journal 2011;Mar 3:342.

7 Brannan SCE, English V, Mussell R, Sheather J and Sommerville A. Ethics briefings. Journal of Medical Ethics 2011 Mar;37(3):190-2.

8 Royal Pharmaceutical Society of Great Britain. From Compliance to Concordance Achieving Shared Goals in Medicine Taking. London: Royal Pharmaceutical Society of Great Britain, 1997.

9 Royal College of General Practitioners. GP Curriculum Statements. London: Royal College of General Practitioners, 2010.

10 Cribb A and Owens J. Whatever suits you: unpicking personalisation for the NHS. Journal of Evaluation in Clinical Practice 2010;16(3):310-14.

11 World Health Organization. Adherence to Long-Term Therapies: Evidence for Action. Geneva: WHO, 2003.

12 Boorse C. Health as a theoretical concept. Philosophy of Science 1977 Dec;44(4):542-73.

13 Daniels N. Just Health Care. Cambridge: Cambridge University Press, 1985.

14 Groopman J. How Doctors Think. New York: Mariner Books, 2008.

15 Canguilhem G. The Normal and the Pathological. New York: Zone Books, 1991.

16 Nordenfelt L. On the Nature of Health: An ActionTheoretic Approach (2e). Dordrecht, The Netherlands: Kluwer, 1995.

17 Seedhouse D. Health: The Foundations for Achievement (2e). Chichester: Wiley, 2001. 
18 Owens $\mathrm{J}$ and Cribb A. Conflict in medical co-production: can a stratified conception of health help? Health Care Analysis 2011 Jul 20.

19 Heath I. A wolf in sheep's clothing: a critical look at the ethics of drug taking. British Medical Journal 2003 Oct;327(7419):856-8.

20 Gawande A. Whose body is it anyway? What doctors should do when patients make bad decisions. Indian Journal of Medical Ethics 2002;10(1).

21 Loewy E. In defense of paternalism. Theoretical Medicine and Bioethics 2005;26(6):445-68.

\section{ADDRESS FOR CORRESPONDENCE}

John Owens

Centre for Public Policy Research

King's College London

London WC2R 2LS

UK

Email: john.owens@kcl.ac.uk

Submitted 9 May 2011, 2 July 2011, accepted for publication 30 September

Read and comment on this article online at www.londonjournalofprimarycare.org.uk/ 